\title{
Exploring New Search Algorithms and Hardware for Phylogenetics: RAxML Meets the IBM Cell
}

\author{
A. STAMATAKIS \\ School of Computer and Communication Sciences, École Polytechnique Fédérale de Lausanne, \\ Lausanne, Switzerland \\ F. BLAGOJEVIC AND D. S. NIKOLOPOULOS \\ Department of Computer Science, Center for High-end Computing Systems, Virginia Tech, \\ Blacksburg, VA, USA \\ C. D. ANTONOPOULOS \\ Department of Computer and Communications Engineering, University of Thessaly, Volos, Greece
}

Received: 8 December 2006; Accepted: 17 March 2007

\begin{abstract}
Phylogenetic inference is considered to be one of the grand challenges in Bioinformatics due to the immense computational requirements. RAxML is currently among the fastest and most accurate programs for phylogenetic tree inference under the Maximum Likelihood (ML) criterion. First, we introduce new tree search heuristics that accelerate RAxML by a factor of 2.43 while returning equally good trees. The performance of the new search algorithm has been assessed on 18 real-world datasets comprising 148 up to 4,843 DNA sequences. We then present the implementation, optimization, and evaluation of RAxML on the IBM Cell Broadband Engine. We address the problems and provide solutions pertaining to the optimization of floating point code, control flow, communication, and scheduling of multi-level parallelism on the Cell.
\end{abstract}

Keywords: phylogenetic inference, maximum likelihood, RAxML, IBM cell

\section{Introduction}

Phylogenetic trees are used to represent the evolutionary history of a set of $n$ organisms. An alignment with the DNA or protein sequences representing $n$ organisms can be used as input for phylogenetic inference. In a phylogeny the organisms of the input data are located at the tips (leaves) of the tree and the inner nodes represent extinct common ancestors. The branches of the tree represent the time which was required for the mutation of one species into another, new one. Phylogenetic trees have many important applications in medical and biological research (see [2] for a summary).
Due to the rapid growth of sequence data over the last years it has become feasible to compute large trees which often comprise more than 1,000 organisms and sequence data from several genes (so-called multi-gene alignments). This means that alignments grow in the number of organisms and in sequence length.

The basic algorithmic problem computational phylogeny faces is the immense amount of alternative tree topologies which grows exponentially with the number of organisms $n$, e.g. for $n=50$ there exist $2.84 * 10^{76}$ alternative trees. In fact, it has only recently been shown that the ML phylogeny problem is NP-hard [6]. In addition, ML-based inference of 
phylogenies is very memory- and floating pointintensive. Since phyloinformatics has definitely entered the HPC era by now, the application of high performance computing techniques as well as the assessment of new CPU architectures can significantly contribute to the reconstruction of larger and more accurate trees. Moreover, typical ML implementations exhibit different levels of parallelism and are thus well-suited as example applications for exploiting unconventional and challenging architectures.

The Cell Broadband Engine (BE) has been developed jointly by Sony, Toshiba and IBM. Although the Cell was originally designed for the set-top box market, it has evolved into a generalpurpose processor for high-performance computing, server and desktop applications. The Cell BE is a heterogeneous multicore processor with nine processing cores: one two-way multithreaded PowerPC Processor Element (PPE) and eight Synergistic Processor Elements (SPEs). The Cell is well suited for data-intensive scientific applications with high demand for memory bandwidth. It offers a unique assembly of MIMD and SIMD execution capabilities and a software-managed memory hierarchy, thus providing ample flexibility in selecting programming and parallelization models for a given application.

According to its specifications [1], the Cell is capable of achieving significant performance improvement over conventional multicore CPUs, including an improved Flops per Watt ratio. However, due to its unconventional architecture, the development of parallel applications that can exploit all advantages of the Cell design, is a challenging task. One of the main difficulties is the management of the local storage of the SPEs by software. Another challenge lies in the distribution of work among SPEs, which can be implemented at multiple degrees of granularity and with a variety of code and data distribution schemes. This paper addresses these problems in the realm of algorithms for phylogenetics and more specifically RAxML [25]. We present optimizations and system software support for vectorization, control flow parallelization, scheduling, and communication on the Cell, along with a stepwise evaluation of these optimizations in RAxML. The optimizations are generic enough to be reused across a wider range of parallel applications.

The remainder of this paper is organized as follows: First, we review related work on IBM Cell portings and tools (Section 2). In Section 3 we provide an overview of RAxML and related work in the area of phylogenetics. We also introduce new tree search heuristics that accelerate RAxML by factor 2.43 while returning equally good trees on realworld datasets of 148 up to 4,843 DNA sequences. The following Section 4 gives a step-by-step description of porting and optimizing RAxML on Cell. The conventional optimizations we used to speedup the execution time include the use of optimized numerical libraries for the SPEs, double-buffering for complete communication/computation overlap, vectorization of floating point operations, and minimization of the ratio of PPE-SPE computation via function off-loading. We also present new Cellspecific optimizations, including the vectorization of conditional statements, asynchronous communication via direct SPE memory accesses and an eventdriven scheduling model, which can selectively exploit coarse-grain and fine-grain parallelism, in response to workload variation. In Section 5 we evaluate performance of RAxML on the IBM Cell and the IBM Power5 multicore processor. We conclude with Section 6.

\section{Related Work on IBM Cell}

Recently, several studies were conducted to measure performance and develop programming models for easier porting as well as optimization of parallel applications on the Cell.

Fatahalian et al. [10] developed Sequoia, a programming language suitable for porting memory-aware applications to machines with different memory hierarchy configurations. One of the target architectures in this study is the Cell Broadband Engine. The authors used Sequoia to port several programs to Cell and obtained memory throughput exceeding $20 \mathrm{~GB} / \mathrm{s}$. Sequoia currently supports applications which can be parallelized via recursive block decomposition, whereas our work focuses on less structured applications that can be parallelized at multiple layers.

Bellens et al. [3] developed a dependence-driven programming model for porting sequential applications to Cell. Their compiler is capable of generating tasks that can potentially be executed in parallel. The supporting runtime system creates a dependence graph of active tasks during execution and determines which tasks can be scheduled for execution on the SPEs. This work considers only one layer of task-level parallelism and does not explore the 
implications of task size and available parallelism within tasks. Both issues are central in the current paper.

Kunzman et al. [17] are in the process of adapting Charm++ on Cell. Charm++ is a runtime system for object-based parallel programming. More specifically, Charm++ is a library of machine-independent object abstractions for scheduling and communication on parallel machines, implemented on both distributed and shared memory systems. No results that would enable comparisons with our work are reported as of the time of writing this paper.

Although Cell has been a focal point in numerous articles in popular press, published research using Cell for real-world scientific applications beyond games and streaming computation is scarce. Besides our study, Hjelte [15] presents an implementation of a smooth particle hydrodynamics simulation on Cell. This simulation requires good interactive performance, since it lies on the critical path of real-time applications such as interactive simulation of human organ tissue, body fluids, and vehicular traffic. Benthin et al. [4] present a parallel ray-tracing algorithm for Cell.

\section{The RAxML Application}

Despite the high computational cost, significant progress has been achieved over the last years in the field of heuristic ML search algorithms with the release of programs such as IQPNNI [19], PHYML [14], GARLI [31] and RAxML [25, 27] to name only a few.

RAxML-VI-HPC (v2.2.0; Randomized Axelerated Maximum Likelihood version VI for High Performance Computing, freely available at http://icwww.epfl.ch/ stamatak) [25] is a program for large-scale ML-based [12] inference of evolutionary trees using multiple alignments of DNA or AA (Amino Acid) sequences. Some of the largest published ML-based phylogenetic analyses to date have been conducted with RAxML $[11,13,21]$. To the best of our knowledge, RAxML has been used to compute trees on the two largest data matrices analyzed under ML to date: a 25,057-taxon alignment of protobacteria (length: 1,463 nucleotides) and a 2,182-taxon alignment of mammals (length: 51,089 nucleotides).

The current version (v2.2.0) implements the new search algorithm described in Section 3.2 as well as the older algorithm (command line switch $-f o$ ) outlined in [25]. A recent performance study [25] on real world datasets with more than 1,000 sequences reveals that it is able to find better trees in less time and with lower memory consumption than other current ML programs (IQPNNI, PHYML, GARLI).

\subsection{Parallel Implementations of ML Programs}

RAxML exploits two levels of parallelism: fine-grain loop-level parallelism and coarse-grain embarrassing parallelism.

RAxML has been parallelized with OpenMP to exploit loop-level parallelism. Like every ML-based program, RAxML exhibits a source of loop-level parallelism in the likelihood functions which typically consume over $90 \%$ of the overall computation time. The OpenMP implementation scales particularly well on large multi-gene alignments due to increased cache efficiency [28].

The MPI version of RAxML exploits the embarrassing parallelism that is inherent to every realworld phylogenetic analysis. In order to conduct such an analysis (see [13] for an example) a number of about 20-200 distinct tree searches (multiple inferences) to find a best-scoring tree on the original alignment as well as a large amount of 100-1,000 bootstrap analyses have to be conducted. Bootstrap Analyses are required to assign confidence values ranging between 0.0 and 1.0 to the inner nodes of the best-known ML tree. This allows to determine how well-supported certain parts of the tree are and is important to draw biological conclusions. Bootstrapping is essentially very similar to multiple inferences. The only difference is that inferences are conducted on a randomly re-sampled alignment (a certain amount of alignment columns is re-weighted) for every bootstrap run. This is performed in order to assess the topological stability of the tree under slight alterations of the input data.

All those individual tree searches, be it bootstrap or multiple inferences are completely independent from each other and can thus be exploited by a simple master-worker scheme. If the dataset is not extremely large, this represents the most efficient approach to exploit HPC platforms for production runs.

Most other parallel implementations of ML programs $[7,19,26,29,31]$ have mainly focused on the intermediate level of parallelism (inference parallelism) which is situated between the loop-level parallelism and coarse-grained parallelism currently 
exploited in RAxML. The related work mentioned above mainly deals with highly algorithm-specific and mostly MPI-based parallelization of various hillclimbing, genetic, as well as divide-and-conquer search algorithms. Finally, Minh et al. [20] recently implemented a hybrid OpenMP/MPI version of IQPNNI which exploits loop-level and inference parallelism.

\subsection{Accelerating the Search Algorithm}

This Section describes a novel heuristic optimization of the RAxML search algorithm that is available as of version 2.2.0. The entire procedure is outlined in Fig. 1.

The fundamental mechanism that is used to search the tree space with RAxML is called Lazy Subtree Rearrangement (LSR, for details see [27]). An LSR consists in pruning/removing a subtree from the currently best tree $t$ and then re-inserting it into all neighboring branches up to a certain distance/radius (rearrangement distance) of $n$ nodes from the pruning point ( $n$ typically ranges from 5 to 25 ). For each possible subtree insertion within the rearrangement distance, RAxML evaluates the log likelihood score of the alternative topology. This is done in a lazy way since only the length of the three branches adjacent to the insertion point/node will be optimized. Thus, an LSR only yields an approximate log likelihood $\operatorname{all}\left(t^{\prime}\right)$ score for each alternative topology $t^{\prime}$ constructed by an LSR from $t$. However, this $\operatorname{all}\left(t^{\prime}\right)$ score can be used to sort the alternative topologies. After this fast pre-scoring of a large number of alternative topologies, only a very small fraction of the best-scoring topologies needs to be optimized more exhaustively to improve the overall tree score. One iteration of the RAxML hill-climbing algorithm consists in performing LSRs on all subtrees of a given topology $t$ for a fixed rearrangement distance $n$. Thereafter, the branches of the 20 best-scoring trees are thoroughly optimized. This procedure of conducting LSRs on all subtrees and then optimizing the 20 best-scoring trees, is performed until no improved tree is encountered.

The main idea of the new heuristics is to reduce the number of LSRs performed. This is done by using an empirical cutoff-rule that stops the recursive descent of an LSR into deeper branches at a higher rearrangement distance from the pruning position if they do not appear to be promising. Thus, if the approximate log likelihood $\operatorname{all}\left(t^{\prime}\right)$ for the current rearranged tree $t^{\prime}$ is worse than the log likelihood $l l(t)$ of the currently best tree $t$ and if the difference $\delta\left(\operatorname{all}\left(t^{\prime}\right), l l(t)\right)$ is larger than a certain threshold $l h_{\text {cutoff }}$ the remaining LSRs below that node are omitted. The threshold $l h_{\text {cutoff }}$ is determined as follows: During the first iteration of the RAxML search algorithm $l h_{\text {cutoff }}=\infty$ which means that no cutoffs are made. In the course of this first iteration, the differences $\delta_{i}\left(\operatorname{all}\left(t_{i}\right), l l(t)\right)$ for all those $i=1 \ldots m$
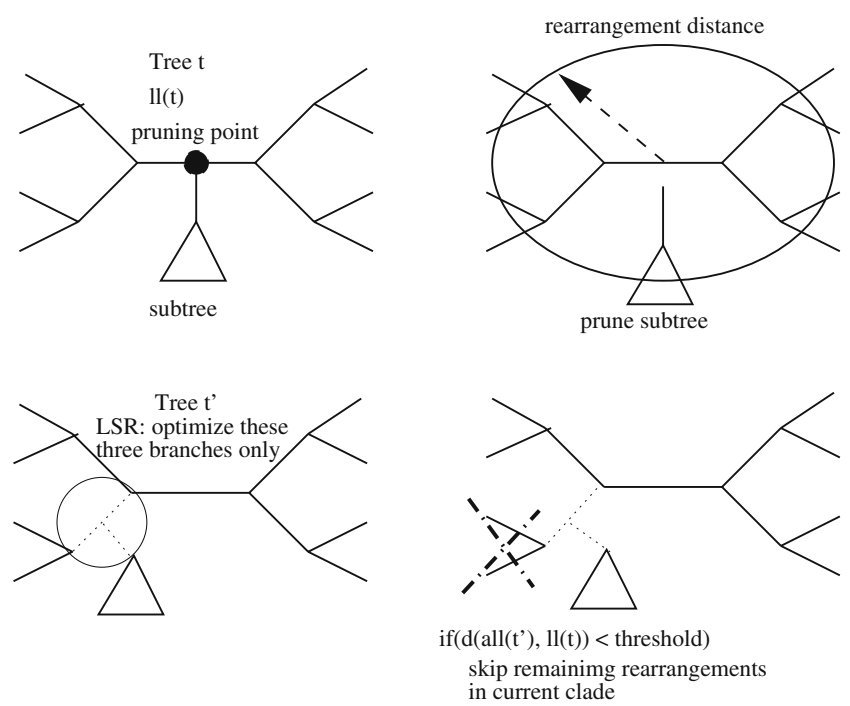

Figure 1. Outline of lazy subtree rearrangements with cutoff procedure. 
alternative tree topologies $t_{i}$ where $\operatorname{all}\left(t_{i}\right) \leq l l(t)$ are stored. The threshold $l h_{\text {cutoff }}$ for the next iteration is set to the average of $\delta_{i}$, i.e. $\sim l h_{\text {cutoff }}=\left(\sum_{i=1}^{m} \delta_{i}\right) / m$. If the search computes an LSR for which $\operatorname{all}\left(t^{\prime}\right) \leq l l(t)$ and $\delta\left(\operatorname{all}\left(t^{\prime}\right), l l(t)\right) \geq l h_{\text {cutoff }}$ it will simply skip the remaining LSRs below the current node. Thus, each iteration $k$ of the search algorithm uses a threshold value $l h_{\text {cutoff }}$ that has been obtained during the previous iteration $k-1$. This allows to dynamically adapt $l h_{\text {cutoff }}$ to the specific dataset and to the progress of the search. The omission of a large amount of unnecessary LSRs that have a high probability not to improve the tree yields substantial run time improvements and returns equally good trees at the same time (see Table 1).

3.2.1. Results. To assess performance of the new heuristics we analyzed 18 real-world datasets comprising 148 to 4,843 sequences from various sources (see Acknowledgment). The computations were performed under the CAT approximation of rate heterogeneity [24], but final tree scores were evaluated with the standard GAMMA model of rate heterogeneity. For each dataset we generated ten starting trees and executed the old and new RAxML search algorithm on each of those starting trees. We performed a total of 380 ML searches which were executed on the Infiniband cluster at the TU München. The cluster is equipped with 36 quadCPU 2.4 GHz AMD Opteron nodes.

Table 1 lists the average final log likelihood values (LH-NEW, LH-OLD) for the old and new versions of the RAxML search algorithm. In addition, it provides the average speedup value per dataset. The average speedup over all datasets is 2.43 . The slight variations in likelihood scores are insignificant.

\section{Porting and Optimizing RAxML on Cell}

\subsection{The Cell BE}

The Cell BE is a heterogeneous multicore processor. The design is inspired by graphics accelerators, which are commonly used in set-top boxes for data streaming computations. In contrast to conventional processors, graphics accelerators typically provide vast memory bandwidth and vectorization capabilities. Therefore, they are able to execute streaming computation kernels such as encryption/decryption, compression, FIR filters and FFTs far more efficiently. The Cell BE integrates eight specialized accelerators with a conventional 64-bit host processor on the same chip. The host processor is a PowerPC SMT core, which runs Linux in a virtualized setting. The PowerPC core communicates via a ring network with eight accelerator cores, called Synergistic Processor Elements, or SPEs. The SPEs and the PPE are laid out in equal distances around a ring called the Element Interconnect Bus (EIB). The EIB can transmit up to 96 bytes per processor cycle, and has a maximum bandwidth of $200 \mathrm{~GB} / \mathrm{s}$. The PPE is also connected to the EIB. An overview of the Cell architecture is provided in Fig. 2.

The SPEs can issue up to two instructions per cycle, one of which can be a 128-bit SIMD instruction. Since the SPEs do not include hardware for branch prediction they rely on software to predict the outcome of branches. In its current implementation, the SPE pipeline is optimized for singleprecision floating-point vector operations, for which the processor can sustain a maximum throughput of over 230 Gflops. This is an artifact of earlier designs of the processor for game consoles, in which double

Table 1. Average final log likelihood values and speedups over ten runs on distinct MP starting trees for 18 real-world datasets.

\begin{tabular}{|c|c|c|c|c|c|c|c|}
\hline No. of Seq. & LH-NEW & LH-OLD & Speedup & No. of Seq. & LH-NEW & LH-OLD & Speedup \\
\hline 148 & $-69,726.05$ & $-69,725.55$ & 1.83 & 150 & $-39,606.06$ & $-39,606.06$ & 2.00 \\
\hline 218 & $-134,195.92$ & $-134,199.30$ & 1.98 & 404 & $-156,151.42$ & $-156,147.25$ & 2.00 \\
\hline 498 & $-219,186.90$ & $-219,186.90$ & 2.21 & 500 & $-85,794.87$ & $-85,794.87$ & 2.11 \\
\hline 628 & $-50,940.81$ & $-50,938.29$ & 2.68 & 714 & $-148,543.21$ & $-148,544.48$ & 2.51 \\
\hline 994 & $-348,936.85$ & $-348,936.85$ & 2.34 & 1,288 & $-395,999.61$ & $-395,999.61$ & 1.92 \\
\hline 1,512 & $-273,435.30$ & $-273,443.51$ & 2.74 & 1,604 & $-167,398.16$ & $-167,399.43$ & 3.31 \\
\hline 1,780 & $-178,930.53$ & $-178,925.02$ & 2.95 & 1,908 & $-149,645.43$ & $-149,645.43$ & 2.89 \\
\hline 2,000 & $-364,916.18$ & $-364,914.44$ & 2.65 & 2,554 & $-318,488.01$ & $-318,488.01$ & 2.49 \\
\hline 4,114 & $-325,621.05$ & $-325,620.54$ & 2.70 & 4,843 & $-748,075.04$ & $-748,067.23$ & 2.48 \\
\hline
\end{tabular}




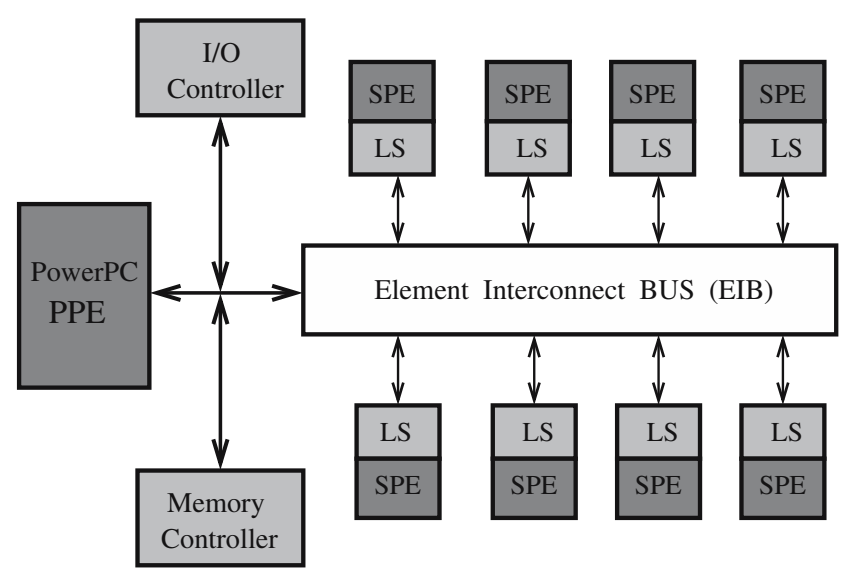

Figure 2. Outline of the CELL BE architecture.

precision floating-point calculations are of lesser importance than in scientific computing. Nevertheless, doubleprecision throughput is still 21 Gflops, which is higher than the throughput of most high-end homogeneous multi-core processors. New versions of the processor for high-performance computing resolve the bottleneck of double-precision floating point operations.

Each SPE on the Cell has 128 128-bit generalpurpose registers and a software-managed fast local storage. The use of a software-managed local storage is unique in the Cell architecture. In the current version of the processor, each SPE has $256 \mathrm{~KB}$ of local storage. This storage is expandable to up to $4 \mathrm{MB}$. Local storage is locally addressable via direct load and store instructions from the owner SPE and globally accessible from other SPEs and the PPE via DMA requests. Software control of the local storage enables the use of customized cache replacement policies on the SPEs. The local storage can also be programmed to operate as a conventional set-associative cache [8].

The SPEs decouple processing from communication, via the use of a memory flow controller (MFC). Each SPE can issue up to 16 concurrent DMA requests, including requests for atomic DMA operations, which can be used in lieu of locks, and requests for scatter-gather memory operations.

\subsection{RAxML Porting Strategy}

We followed a three-step process for porting RAxML to Cell:

1. We identified and off-loaded the compute intensive parts of RAxML to the SPEs.
2. We optimized the off-loaded code on the SPEs. We used optimized numerical libraries for the SPEs, double buffering, vectorization of computation, vectorization of control flow, and PPESPE communication optimizations.

3. Finally, we developed an event-driven scheduler for RAxML's nested parallel code, along with the necessary system software support.

The first two steps follow the function off-loading programming model, which arguably provides the easiest path for porting MPI applications on the Cell $[8,9]$. The last step is a new contribution to support the execution of parallel programs with multiple levels of parallelism on the Cell.

In the following Sections we describe the porting and optimization process, together with speedups obtained at each optimization step.

\subsection{Porting the MPI Code}

The version of RAxML that we ported on Cell is based on the new, improved search heuristics, described in Section 3.2. We initially executed the MPI version of RAxML on the PPE. Since the PPE is a dual-threaded processor, the PPE can execute two MPI processes simultaneously. These processes share the resources of the PPE, except from the register file. Therefore, their parallel execution on the PPE is not expected to scale as well as on a twoway SMP. Although it is natural to consider using the PPE for direct porting of MPI code without modifications, this approach is clearly suboptimal. Due to the heterogeneity of the Cell, further steps are 
needed to off-load and optimize most, if not all, of the computation within each MPI process, in order to exploit the computational capacity of the SPEs.

We explored two strategies for off-loading code on the Cell SPEs. The first strategy is pure task-level off-loading. Each MPI process running on the PPE off-loads functions on the SPEs and each function executes from start to finish on the SPE it is assigned to. The second strategy is a hybrid task-level and loop-level parallelization scheme. Functions are offloaded from the MPI process to some of the SPEs. The remaining SPEs are used for work sharing of parallel loops included in the already off-loaded functions. These parallelization strategies are described further in Section 4.7.

\subsection{Selecting Functions for Off-loading}

In order to find the functions in RAxML that are suitable for SPE execution, we profiled the code using gprof. For all the profiling and benchmarking runs of RAxML presented in this paper, we use the input file 42 SC, which contains 42 organisms, each represented by a DNA sequence of 1,167 nucleotides. The number of distinct data patterns in a DNA alignment is in the order of 250. Profiling the sequential version of RAxML on the IBM Power5 processor shows that $96.6 \%$ of the execution time is spent in four functions:

- $64.62 \%$ of the time is spent in the function newview () . This function computes the partial likelihood vector [12] at an inner node of the phylogenetic tree.

- $26.88 \%$ of the time is spent in makenewz () . This function optimizes the length of a given branch with respect to the tree likelihood using the Newton-Raphson method.

- $2.87 \%$ of the time is spent in newviewPartial(), which optimizes the per-site evolutionary rates for the GTRCAT approximation (see [24] for details).

- Finally, $2.29 \%$ of the time is spent in evaluate(). This function calculates the Log Likelihood score of the tree at a given branch by summing over the partial likelihood vector entries. Note that the log likelihood value is the same at all branches of the tree if the model of nucleotide substitution is timereversible [12].

The prerequisite for computing evaluate() and makenewz () is that the likelihood vectors at the nodes to the right and left of the branch have been computed. Thus, makenewz () and evaluate () initially make calls to newview() before they can execute their own computation. The newview() function at an inner node $\mathbf{p}$ calls itself recursively when the two children $\mathbf{r}$ and $\mathbf{q}$ are not tips (leaves) and the likelihood array for $\mathbf{r}$ and $\mathbf{q}$ has not already been computed. Consequently, the first candidate for off-loading is newview(). Although makenewz () and evaluate () are both taking a smaller portion of execution time than newview(), off-loading these two functions can also lead to significant speedup (see Section 4.6). Besides the fact that each function can be optimized and executed faster on an SPE, having all four functions off-loaded to an SPE significantly reduces the amount of PPESPE communication.

The code executed on the SPE has to be compiled separately (using the SPE specific compiler) from the code executed on the PPE. We choose to include all four off-loaded functions in one code module. This approach has the advantage of having all off-loaded functions in the SPE's local storage during the entire execution of the program. Consequently, an offloaded function can be invoked without introducing the overhead of moving its code from global memory to local storage. However, this decision imposes a trade-off, since the extended code segment in the SPE local storage reduces the available space for the heap and stack segments. In the case of RAxML, the total size of all four off-loaded functions is $110 \mathrm{~KB}$. The remaining space (146 KB) is large enough to store the heap and stack segments of each of the four functions, as well as the buffers needed for communication-computation overlap.

To keep the implementation simple, the call to each off-loaded function in the original MPI code is executed with the same signature on the PPE in the Cell code. We replaced the original body of each function with communication code needed to transfer local data used in the function from the PPE to SPEs. Whenever an off-loaded function is called, the PPE sends a signal to the SPE thread and waits for the SPE thread to complete the function and return the result. While waiting for the SPE code to finish, the PPE seeks functions for off-loading from other MPI processes. This process is described in more detail in Section 4.7.

All four off-loaded functions are executed inside a single SPE thread. The SPE thread is created at the 
beginning of the program and stays alive during the entire program execution. Depending on the content of a triggering signal received from the PPE, the thread executes one of the four off-loaded functions. By having a single thread active during the entire program execution, we avoid excessive overhead from repeated spawning and joining of threads. The SPE threads execute a busy wait for a PPE signal.

Data consistency is maintained at the granularity of off-loaded functions. Each function is individually responsible for collecting local updates and propagating these updates to global shared memory.

\subsection{Optimizing Off-loaded Functions by Example of newview ()}

Since newview () is the most computationally expensive function in the code, it becomes the first candidate for optimization. We found that naïvely off-loading newview () slows down the sequential version of the code by a factor of 2.8. Therefore, exhaustive optimization of the function on the SPE was necessary. Table 2 summarizes the execution times of RAxML before and after newview() is offloaded. The first column shows the number of workers (MPI processes) used in the experiment and the amount of work done (number of bootstraps).

We profiled the function newview () using the decrementer register of the SPE. We identified four code segments that consume almost the entire execution time in newview () :

1. Math library functions, such as $\exp ()$ and $\log ()$. These functions are very expensive if their native implementations are executed on the SPE. In newview () , the $\exp ()$ function is used to compute the transition probabilities of the nucleotide substitution matrix for the branches from the root of a subtree to its descendants. The $\log ()$ function is used to scale the branch lengths for numerical reasons [23].

2. An if (..) statement in the code which determines the value of a conjunction of four expressions is the second bottleneck. This conditional statement is used to check if small likelihood vector entries need to be scaled to avoid numerical underflow (similar operations are used in every ML implementation).

3. Blocking DMA requests also have a significant impact on execution time. Whenever data re-
Table 2. Execution time of RAxML (in seconds).

\begin{tabular}{ll}
\hline & Time (s) \\
\hline (a) & \\
1 worker, 1 bootstrap & 28.3 \\
2 workers, 8 bootstraps & 152.56 \\
2 workers, 16 bootstraps & 309.53 \\
2 workers, 32 bootstraps & 622.43 \\
(b) & \\
1 worker, 1 bootstrap & 80.52 \\
2 workers, 8 bootstraps & 348.36 \\
2 workers, 16 bootstraps & 696.12 \\
2 workers, 32 bootstraps & $1,375.09$ \\
\hline
\end{tabular}

The input file is $42 \_S C$ : (a) The application is executed on the PPE, (b) newview (), without optimizations, is off-loaded to one SPE.

quired for the computation is not in local storage, the program has to wait for the necessary data to be fetched from global memory.

4. All double precision floating point arithmetic used for the likelihood vector calculation is not natively vectorized or optimized.

In the next few subsections we describe performance optimizations for the off-loaded newview () function. We applied analogous optimizations to the remaining off-loaded functions. We do not discuss the other functions in more detail due to space limitations.

4.5.1. Mathematical Functions. The first step in reducing the execution time of the off-loaded function was to replace the expensive math functions $\exp ()$ and $\log ()$ with the mathematical functions provided by the Cell SDK 1.1. The $\exp ()$ and $\log ()$ functions provided by the Cell SDK are implementations of numerical methods for exponent and $\log$ arithm calculation. The $\exp ()$ and $\log ()$ functions represent less than $1 \%$ of the total number of floating point operations executed in the off-loaded function, however they account for $56 \%$ of execution time in newview () . Using the implementations of the exponent and logarithm functions provided by the Cell SDK improves total execution time by $37-41 \%$. Table 3 shows the execution times of the four test runs of RAxML after newview() is off-loaded and $\exp ()$ and $\log ()$ functions are replaced with 
Table 3. Execution time of RAxML with no off-loading (a), and with newview() off-loaded and optimized to use numerical functions from the SDK library.

Time (s)

(b)

$\begin{array}{ll}1 \text { worker, } 1 \text { bootstrap } & 47.94 \\ 2 \text { workers, } 8 \text { bootstraps } & 218.17 \\ 2 \text { workers, } 16 \text { bootstraps } & 433.94 \\ 2 \text { workers, } 32 \text { bootstraps } & 871.49\end{array}$

The input file is 42_SC.

optimized implementations. Notice that the offloaded code is still $40-47 \%$ slower than the nonoff-loaded code.

4.5.2. Optimizing Conditional Statements. Function newview () is always invoked at an inner node of the tree (p) which is at the root of a subtree. The main computational kernel of newview() has a switch statement which selects one out of four paths of execution. If one or both descendants $\mathbf{r}$ and $\mathbf{q}$ of $\mathbf{p}$ are tips (leaves) the computations of the main for-loop in newview() can be simplified. This optimization leads to significant performance improvements [25]. Thus, there are distinct implementations of the main computational part of newview () for the case that $\mathbf{r}$ and $\mathbf{q}$ are tips, $\mathbf{r}$ is a tip, $\mathbf{q}$ is a tip, or $\mathbf{r}$ and $\mathbf{q}$ are both inner nodes.Each path in the switch statement leads to a large loop which performs the likelihood vector calculations. Each iteration of the large loop executes a large if () statement which determines the value of a conjunction of four arithmetic expressions. This conditional statement checks if likelihood scaling is required to prevent numerical underflow. On an SPE, a mispredicted branch incurs a penalty of 20 cycles (IBM, Cbe_tutorial_v1.1, 2006). Using the decrementer register to profile the off-loaded code, and after optimization of $\exp ()$ and $\log ())$, we find that $39 \%$ of the execution time of newview () is spent in checking the condition of the if () statement executed in the likelihood vector calculation loop. The conditional statement is shown in Fig. 3. minlikelihood is a positive constant and all operands are double precision floating point values.

The $\mathbf{A B S}()$ function increases the number of condition checking (each $\mathrm{ABS}()$ function executes an additional comparison), therefore the number of conditions that need to be checked in this statement is actually eight.

On an SPE, two integer numbers can be compared significantly faster than two double precision floating point numbers. The advantage of integers is that they can be compared using the existing SPE intrinsics. Current SPE intrinsics support comparison of at most 32-bit integer values. Sixty-four bit integers can also be compared relatively fast, by combining 32-bit integer intrinsics. The spu-gcc compiler automatically optimizes conditional statements that operate on integer values, by replacing them with suitable SPE intrinsics.

According to the IEEE standard, all double precision floating point numbers are "lexicographically ordered." In other words, if two double floating point numbers $a$ and $b$ are ordered $(a<b)$, then their bit patterns will be ordered in the same way when interpreted as SignMagnitude integers [16] (*(unsigned long long*)a $<*($ unsigned long long $*) b)$. However, this rule can only be applied if both numbers are greater than 0 . In the conditional statement that we are trying to optimize all parameters are greater than 0 (we know that minlikelihood is a constant greater than 0). Therefore, instead of comparing double precision floating point values, we can optimize the problematic if () statement by casting all our operands to unsignedlonglong before comparing them. To avoid the branch used in the $\mathbf{A B S}()$ function, we transform all our operands to positive numbers, using a bitwise AND.

This optimization reduces the time spent in the conditional statement to only $7 \%$ of the execution time of newview () . The total execution time of RAxML is reduced by $20 \%$. Table 4 shows the new execution times of RAxML after optimizing both

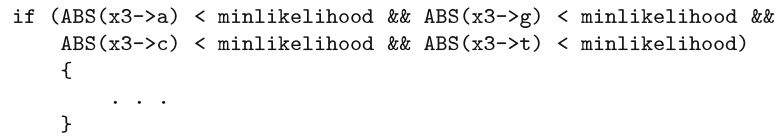

Figure 3. Conditional statement which takes $39 \%$ of newview() execution time, after optimization of numerical functions. 
Table 4. Execution time of RAxML after the floating-point conditional statement is transformed to an integer conditional statement.

\begin{tabular}{ll}
\hline & Time (s) \\
\hline (b) & \\
1 worker, 1 bootstrap & 38 \\
2 workers, 8 bootstraps & 177.68 \\
2 workers, 16 bootstraps & 354.85 \\
2 workers, 32 bootstraps & 703.95 \\
\hline
\end{tabular}

The input file is $42 \_$SC.

conditional statements and numerical functions in newview () .

4.5.3. Double Buffering and Memory Management. The number of iterations in the main loop of newview () depends on the input alignment length. This loop operates on three arrays with equal length (likelihood vector at current subtree root, and likelihood vectors at left and right child). The loop has no loop-carried dependencies and executes as many iterations as the length of the three arrays. Since the size of the local storage is only $256 \mathrm{~KB}$, the arrays cannot be stored permanently in local storage. Instead, the arrays are strip-mined and processed in blocks. The block size is selected such that the computation on a block can overlap completely with the memory latency for fetching the next block. We use a $2 \mathrm{~KB}$ buffer for caching each array block, which holds enough data to execute 16 loop iterations. We also use double buffering to mask memory latency, which amounts to $8 \%$ of the execution time of the code before optimization. Table 5 shows the improved execution times of RAxML after optimization of numerical operations, optimization of conditionals and memory latency overlap, which improved total execution time by $3-5 \%$.

4.5.4. Vectorization. The core of the computation in newview () is concentrated in two loops. The first loop is executed at the beginning of the function and computes the individual transition probability matrices for each distinct rate category of the CAT model [24]. The number of iterations is relatively small (between 1 and 25) and each iteration executes 36 double precision floating point operations. The second loop calculates the likelihood vector. The length of this vector corresponds to the number of distinct alignment patterns. Each iteration of this loop executes 44 double precision floating point operations for the CAT model.

The kernel of the first loop in newview() is shown in Fig. 4a. In Fig. 4b we show the same code vectorized for the SPE. The function spu_mul() multiplies two vectors (in this case the arguments are vectors of doubles). Function _exp_v () is the vector version of the exponential function mentioned in Section 4.5.1. After vectorization, the number of floating point operations executed in the body of the first loop drops from 36 to 24. An additional instruction is required to create a vector from a scalar element. Due to pointer arithmetic on dynamically allocated data structures, automatic vectorization of this code would be particularly challenging for a compiler.

The second loop is vectorized in a similar way. Figure 5 shows the core of the second loop before and after vectorization. The variables $\mathbf{x 1}->\mathbf{a}, \mathbf{x 1}->\mathbf{c}$, $\mathbf{x 1}->\mathbf{g}, \mathbf{x 1}->\mathbf{t}$, belong to the same $\mathrm{C}$ structure (likelihood_vector) and are stored in contiguous memory locations. In Fig. 5a we see that only three of these variables are multiplied with the elements of array left. Therefore, vectorization cannot be accomplished by simply interpreting the members of the likelihood_vector structure that reside in consecutive memory locations as vectors. We actually need to create vectors using special intrinsics for vector creation, such as spu_splats () .

Vectorization decreases the execution time of the two major loops in newview () from 12.8 to $7.3 \mathrm{~s}$. Table 6 summarizes the execution times for RAxML, after vectorization, optimization of numerical functions, optimization of conditional statements and optimization for memory latency overlap in

Table 5. Execution time of RAxML with double buffering applied to overlap DMA transfers with computation, after optimization of numerical functions and conditionals.

\begin{tabular}{ll}
\hline & Time (s) \\
\hline 1 worker, 1 bootstrap & 36.29 \\
2 workers, 8 bootstraps & 169.50 \\
2 workers, 16 bootstraps & 338.24 \\
2 workers, 32 bootstraps & 688.04 \\
\hline
\end{tabular}

The input file is 42_SC. 


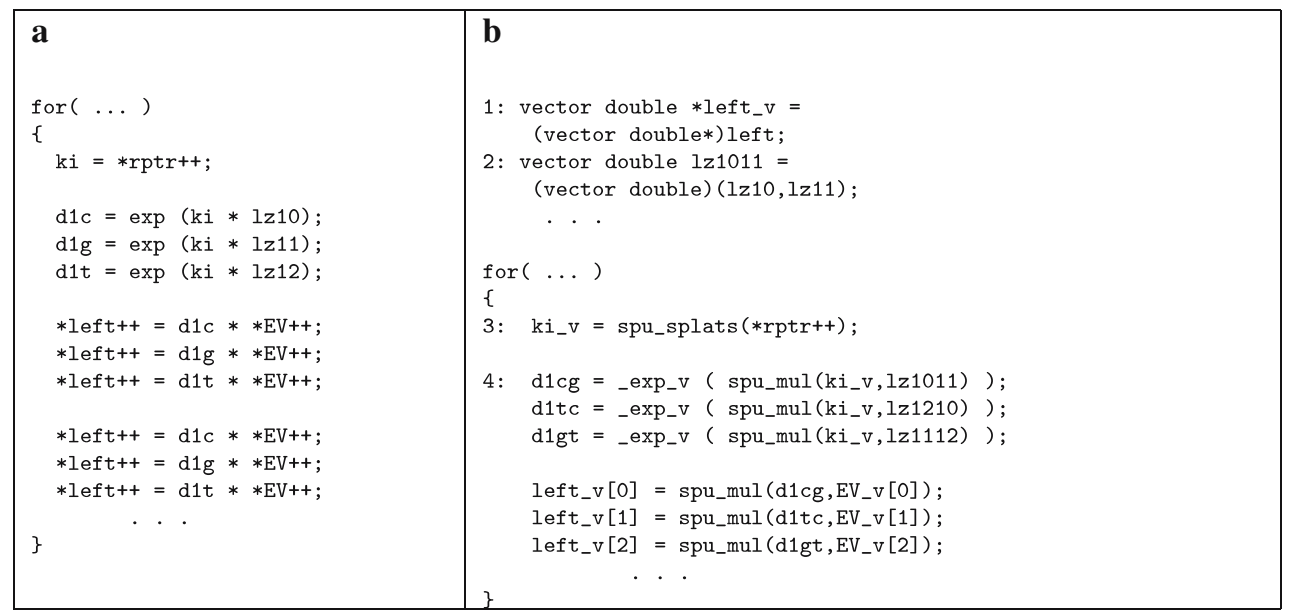

Figure 4. The body of the first loop in newview(): a Non-vectorized code, b Vectorized code.

newview () . After vectorization, the code which offloads newview () on the SPE becomes up to $7 \%$ faster than the code without off-loading.

4.5.5. PPE-SPE Communication. Although newview() dominates the execution time of RAxML, each instance of the function executes for only $82 \mu \mathrm{s}$ on average. This means that the granularity of the function is small and function invocation overhead when the function is off-loaded on the SPE is by no means negligible, since it requires PPE to SPE communication. For our test dataset, the function is called 178,244 times.

We originally implemented PPE to SPE communication using mailboxes, which offer a programmable, high-level interface for communication via message queues. After further experimentation, we found that that DMA transfers achieved lower latency than using mailboxes and improved application execution time by a further $2-11 \%$ in our test cases. Table 7 shows the execution times of RAxML when DMA transfers are used instead of mailboxes and after all optimizations described so far. The input dataset is $42 \_$SC.

It is interesting to note that direct memory-tomemory communication is an optimization which scales with parallelism on Cell, i.e. the impact on performance improves as the code uses more SPEs. As the number of workers and bootstraps executed on the SPEs increases, the code becomes more communication-intensive, due to the fine granularity of the off-loaded functions. Fast communication by DMA therefore becomes critical.

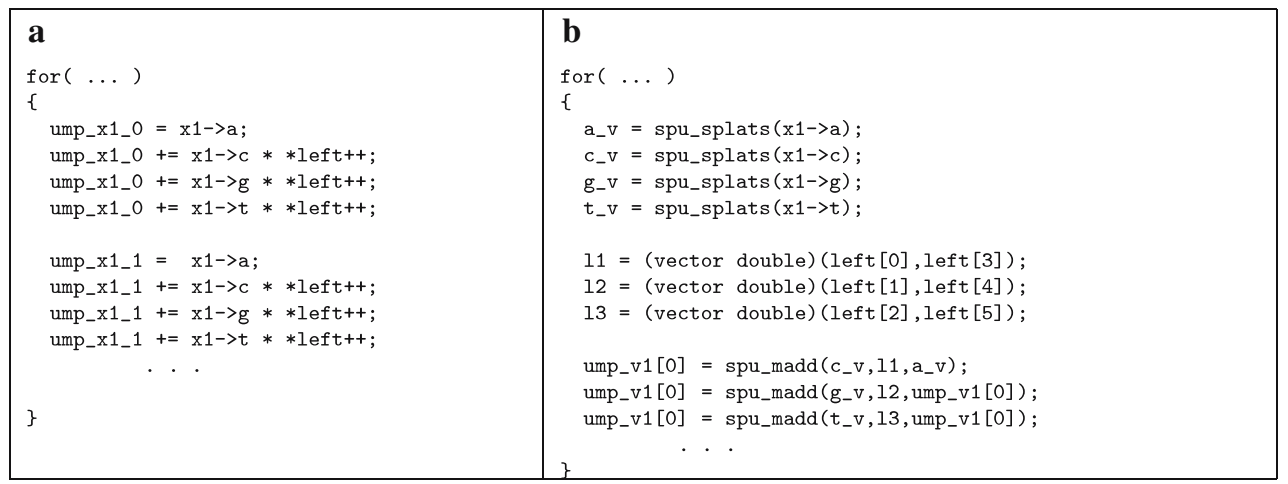

Figure 5. Core of likelihood calculation loop in newview(): a Non-vectorized code, b Vectorized code. 
Table 6. Execution time of RAxML after vectorization, optimization of numerical functions, optimization of conditionals and optimization for memory overlap.

\begin{tabular}{ll}
\hline & Time (s) \\
\hline 1 worker, 1 bootstrap & 30.13 \\
2 workers, 8 bootstraps & 143.20 \\
2 workers, 16 bootstraps & 287.31 \\
2 workers, 32 bootstraps & 577.1 \\
\hline
\end{tabular}

The input file is $42 \_S C$.

\subsection{Off-loading Remaining Functions}

After off-loading and optimizing the newview() function, we proceeded with off-loading the three remaining compute-intensive functions: makenewz () , evaluate(), and newviewPartial().

Off-loading makenewz () and evaluate () was straightforward. We repeated the same off-loading procedure and analogous optimizations as with newview () . As described in Section 4.4, all offloaded functions are written to the same SPE code module. In this way the off-loaded code remains in the local storage during the entire execution of the application. Consequently, we avoid the cost of repeatedly loading different code modules into local storage. Moreover, we reduce PPE-SPE communication, since each invocation of newview() by either makenewz () or evaluate(), can be performed locally on an SPE without involving the PPE.

Off-loading newviewPartial() was a more challenging task. This function is used to re-compute the per-site $\log$ likelihood values $l l_{i}$ at column $i$ of the alignment given a rate $r_{i}$ during the per-site evolutionary rate optimization process (see [24] for details). Since optimizing the $r_{i}$ implies changes of the likelihood vector values at position $i$ in the entire tree, a complete tree traversal (as opposed to a partial tree traversal induced by LSRs as described in Section 3.2) must be carried out to obtain the $l l_{i}$ for $r_{i}$ at each alignment position $i$. Some changes in the data structures were required to allow for future parallel execution of newviewPartial(). Instead of using the likelihood vector arrays allocated for computations over the whole alignment length with newview () , makenewz () , and evaluate () each recursive invocation of newviewPartial () uses the heap to create a private local likelihood vector of length one. These changes enable the concurrent computation of individual per-site log likelihood values $l l_{i}, l l_{j}$ at distinct alignment positions $i \neq j$ with different rates $r_{i}, r_{j}$.

Due to the fine granularity of newviewPartial(), which only operates on one single alignment position and the high amount of calls per alignment position (the $r_{i}$ are optimized in a Brent-like procedure [24]) we off-loaded the large for-loop that optimizes all $r_{i}$ in optimizeRateCategories() to the SPE. This large for-loop has already been parallelized in the current OpenMP version of RAxML using the OpenMP schedule(dynamic) clause. Dynamic scheduling avoids potential load imbalance because the optimization of each $r_{i}$ requires a different number of iterations until convergence. The parallelization of newviewPartial() with OpenMP yielded a performance improvement of $16 \%$ on the 404 sequence dataset with 7,429 distinct patterns from Table 1 on a four-way AMD Opteron. This large performance improvement is due to the fact that newviewPartial() consumes a significantly larger part of execution time for long multi-gene alignments.

With all four functions off-loaded and optimized on the SPE, the application was performing 34-36\% faster. Compared to the initial code which is entirely executed on the PPE, the optimized code is $29 \%$ faster. When more than one MPI processes is used and more than one bootstrap is off-loaded to SPEs, the gains from off-loading reach $47 \%$. Table 8 summarizes the execution times for RAxML when all four functions are off-loaded and optimized for the 42_SC dataset.

\subsection{Scheduling Multilevel Parallelism}

The distribution of resources on the Cell often introduces an imbalance between computation sup-

Table 7. Execution time of RAxML after optimizing communication to use DMA transfers and all previously described optimizations.

\begin{tabular}{ll}
\hline & Time (s) \\
\hline 1 worker, 1 bootstrap & 29.9 \\
2 workers, 8 bootstraps & 126.87 \\
2 workers, 16 bootstraps & 253.67 \\
2 workers, 32 bootstraps & 514.89 \\
\hline
\end{tabular}

The input file is 42_SC. 
Table 8. Execution time of RAxML after off-loading and optimizing four functions: phvnewview(), phvmakenewz(), phvevaluate() and phvevaluatePartial().

\begin{tabular}{ll}
\hline & Time (s) \\
\hline 1 worker, 1 bootstrap & 20.4 \\
2 workers, 8 bootstraps & 84.41 \\
2 workers, 16 bootstraps & 165.3 \\
2 workers, 32 bootstraps & 330.62 \\
\hline
\end{tabular}

The input file is $42 \_$SC.

ply and demand. The processor has eight SPEs, however, at most two threads can off-load code from the PPE to the SPEs at the same time. To overcome this limitation, we explored an event-driven programming model and the associated system software support. In this model, we allow an arbitrary number of MPI processes to share the PPE and implement a context switching strategy which opts for switching the context of a PPE thread upon off-loading a function from the current context, in anticipation of more opportunities for function offloading in other contexts. The intuition is that offloading a function from a PPE thread is typically followed by idle time on the PPE thread, which can be overlapped with computation originating in other PPE threads.

We have extended our event-driven scheduling model with an algorithm which tracks SPE utilization and assigns SPEs to off-loaded functions based on utilization. More specifically, each function receives one or more SPEs, which can be used for parallelization of loops enclosed in the function, with mechanisms and policies similar to those of OpenMP. Parallelization across SPEs assumes that the work is distributed to the worker SPE threads involved in the computation. All the communication among the SPE threads is carried out via their local storages. When they are created, all SPE threads exchange the base addresses of their local storages (this is done through the PPE). As a result, each SPE thread owns a structure where it keeps the base addresses of the local storages of all other threads involved in the computation. A thread running on an SPE communicates to other SPE threads by issuing DMA requests to their private local storages.

The assignment of SPEs to functions depends on the number of SPEs which are idling during a predefined interval of recent execution. More details on our event-driven model are provided in [5].
The new scheduling model reduces the execution time of one bootstrap by $36 \%$, compared to our original static off-loading scheme, with all SPEspecific optimizations integrated in the code. The reason for the high speedup is the ability to distribute loops inside off-loaded functions across SPEs. When loop parallelism and task parallelism are exploited simultaneously in off-loaded functions, the execution time is reduced by up to $63 \%$. Table 9 summarizes the execution times of the optimized implementation of RAxML with our event-driven programming and scheduling model.

\section{Comparison with the IBM Power5}

It is useful to compare the performance of the Cell against other multicore processors, since such a comparison provides valuable insight both for application developers working on adapting their software to emerging computer architectures, and to computer architects who are looking into improving their hardware to address the needs of challenging applications.

In this Section we compare the performance of Cell against an IBM Power5, using our test runs of RAxML. The IBM Power5 is a homogeneous dualcore processor, where each core is itself a two-way simultaneous multithreaded processor. Porting the MPI version of RAxML to the Power5 is straightforward, since all that needs to be done is load four MPI processes (workers) on the four execution contexts of the processor. The Power5 used for this experiment runs at $1.65 \mathrm{GHz}$, and has $32 \mathrm{~KB}$ of L1$\mathrm{D}$ and L1-I cache, $1.92 \mathrm{MB}$ of L2 cache and $36 \mathrm{MB}$ of L3 cache.

Figure 6 provides the execution times on the two processor types for up to 128 bootstraps, a scale

Table 9. Execution time of RAxML with the event-driven programming and scheduling model (MGPS) is used.

\begin{tabular}{ll}
\hline & Time (s) \\
\hline 1 bootstrap & 14.1 \\
8 bootstraps & 26.7 \\
16 bootstraps & 53.63 \\
32 bootstraps & 107.2 \\
\hline
\end{tabular}

The input file is 42_SC. The number of workers is variable and is selected at runtime by the scheduler. 


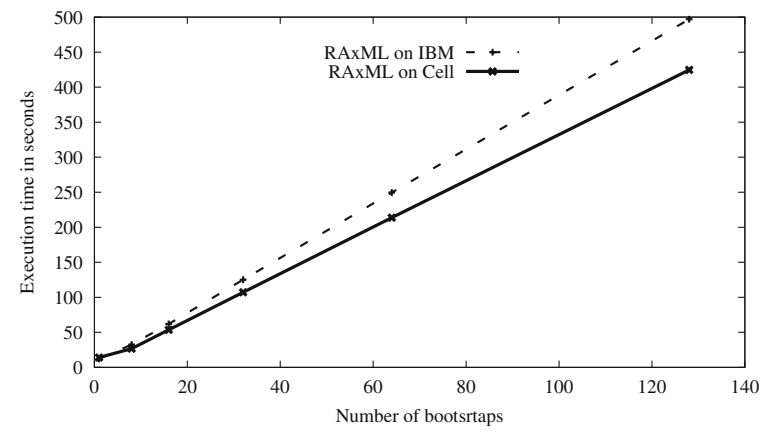

Figure 6. RAxML performance on a multi-core IBM Power5 and the IBM Cell. The number of bootstraps performed covers 1 , $8,16,32,64$ and 128 runs.

which is more representative of real-world data sets for RAxML. The Cell outperforms the IBM Power5 by $15 \%$ on average. Although the difference seems small, a number of considerations should be taken into account. The Power5 has a lower clock frequency, but significantly more secondary and tertiary cache space available to each core. Furthermore, double-precision floating point arithmetic is unoptimized on the Cell's SPE pipelines, leading to a markedly large reduction in processor throughput (up to a factor of 10) compared to single-precision floating point arithmetic. Furthermore, hardware studies of the Cell indicate that the processor is significantly more power-efficient than the Power5, claiming nominal power consumption in the range of 27-43 W for the $3.2 \mathrm{GHz}$ model used in this study [30], as opposed to a reported $150 \mathrm{~W}$ for the Power5 [18]. Taking these observations into consideration, we conclude that the Cell provides a leap forward in performance compared to homogeneous, generalpurpose multicore processors. Our study is the first to demonstrate this leap using complex, non-trivial parallel code from the field of computational biology.

\section{Conclusion and Future Work}

We presented an improved heuristic search algorithm for RAxML as well as a detailed step-by-step description of porting RAxML to IBM Cell. The incremental parallelization and optimization methodology presented in this paper can serve as a guideline for parallelization of non-trivial applications on Cell. The optimizations and system software for multilevel parallelization introduced in this paper resolve bottle- necks which are common to many applications and even multicore architectures other than the Cell.

The porting strategy and methods developed for exploiting fine-grain parallelism in RAxML on the Cell are generally applicable to a broad range of programs for ML-based (GARLI [31], IQPNNI [19], PHYML [14]) and Bayesian (MrBayes [22]) phylogenetic inference. All these programs spend $90-95 \%$ of their total execution time for the evaluation of the likelihood function and face similar problems with respect to memory transfer and function optimization. The strategies and scheduling techniques for coarse-grain parallelism are-with some modifications-also applicable to the MPI-versions of GARLI and IQPNNI. In fact, there already exist a hybrid MPI/OpenMP version of IQPNNI [20] and an OpenMP parallelization of PHYML (Michael Ott, personal communication).

Future work will focus on improved ways to handle recursions on SPEs which will allow for inference of large real-world datasets.

\section{Acknowledgments}

We would like to thank Olaf Bininda-Emonds, Nicolas Salamin, Josh Wilcox, Daniel Dalevi, Usman Roshan, Chuck Robertson, and Markus Göker for letting us use their often tediously hand-aligned sequence data to assess RAxML performance.

\section{References}

1. IBM, "Cell broadband engine programming tutorial version 1.0," Available at: http://www-106.ibm.com/developerworks/ eserver/library/es-archguide-v2.html.

2. D. A. Bader, B. M. E. Moret, and L. Vawter, "Industrial Applications of High-performance Computing for Phylogeny Reconstruction," in Proc. of SPIE ITCom, vol. 4528, 2001, pp. 159-168.

3. P. Bellens, J. M. Perez, R. M. Badia, and J. Labarta, "Cells: A Programming Model for the Cell be Architecture," in Proc. of SC2006, November 2006.

4. C. Benthin, I. Wald, M. Scherbaum, and H. Friedrich, "Ray Tracing on the CELL Processor," Technical Report, inTrace Realtime Ray Tracing GmbH, No inTrace-2006-001, 2006.

5. F. Blagojevic, D. S. Nikolopoulos, A. Stamatakis, and C. D. Antonopoulos, "Dynamic Multigrain Parallelization on the Cell Broadband Engine," in Proc. of PPoPP 2007, San Jose, CA, March 2007. 
6. B. Chor and T. Tuller, "Maximum Likelihood of Evolutionary Trees: Hardness and Approximation," Bioinformatics, vol. 21, no. 1, 2005, pp. 97-106.

7. Z. Du, F. Lin, and U. Roshan, "Reconstruction of Large Phylogenetic Trees: A Parallel Approach," Computational Biology and Chemistry, vol. 29, no. 4, 2005, pp. 273-280.

8. A. E. Eichenberger et al., "Optimizing Compiler for a Cell processor," Parallel Architectures and Compilation Techniques, September 2005.

9. D. Pham et al., "The Design and Implementation of a First Generation Cell Processor," Proc. Int'l Solid-State Circuits Conf. Tech. Digest, IEEE Press, 2005, pp. 184-185.

10. K. Fatahalian et al., "Sequoia: Programming the Memory Hierarchy," in Proc. of SC2006, November 2006.

11. R. E. Ley et al., "Unexpected Diversity and Complexity of the Guerrero Negro Hypersaline Microbial Mat," Appl. Environ. Microbiol., vol. 72, no. 5, 2006, pp. 3685-3695, May.

12. J. Felsenstein, "Evolutionary Trees from DNA Sequences: A Maximum Likelihood Approach," J. Mol. Evol., vol. 17, 1981, pp. 368-376.

13. G. W. Grimm, S. S. Renner, A. Stamatakis, and V. Hemleben, "A Nuclear Ribosomal DNA Phylogeny of Acer Inferred with Maximum Likelihood, Splits Graphs, and Motif Analyses of 606 Sequences," Evolutionary Bioinformatics Online, vol. 2, 2006, pp. 279-294.

14. S. Guindon and O. Gascuel, "A Simple, Fast, and Accurate Algorithm to Estimate Large Phylogenies by Maximum Likelihood," Syst. Biol., vol. 52, no. 5, 2003, pp. 696-704.

15. N. Hjelte, Smoothed Particle Hydrodynamics on the Cell Broadband Engine. Masters Thesis, June 2006.

16. W. Kahan, "Lecture Notes on the Status of IEEE Standard 754 for Binary Floating-point Arithmetic," in IEEE, 1997.

17. D. Kunzman, G. Zheng, E. Bohm, and L. V. Kalé, "Charm++, Offload API, and the Cell Processor," in Proc. of the Workshop on Programming Models for Ubiquitous Parallelism, Seattle, WA, USA, September 2006.

18. Sun Microsystems, Sun UltraSPARC T1 Cool Threads Technology, December 2005. http://www.sun.com/aboutsun/ media/presskits/networkcomputing05q4/T1Infographic.pdf.

19. B. Q. Minh, L. S. Vinh, A. V. Haeseler, and H. A. Schmidt, "pIQPNNI: Parallel Reconstruction of Large Maximum Likelihood Phylogenies," Bioinformatics, vol. 21, no. 19, 2005, pp. 3794-3796.

20. B. Q. Minh, L. S. Vinh, H. A. Schmidt, and A. V. Haeseler, "Large Maximum Likelihood Trees," in Proc. of the NIC Symposium 2006, 2006, pp. 357-365.

21. C. E. Robertson, J. K. Harris, J. R. Spear, and N. R. Pace, "Phylogenetic Diversity and Ecology of Environmental Archaea," Curr. Opin. Microbiol., vol. 8, 2005, pp. 638-642.

22. F. Ronquist and J. P. Huelsenbeck, "MrBayes 3: Bayesian Phylogenetic Inference under Mixed Models," Bioinformatics, vol. 19, no. 12, 2003, pp. 1572-1574.

23. A. Stamatakis, Distributed and Parallel Algorithms and Systems for Inference of Huge Phylogenetic Trees based on the Maximum Likelihood Method, $\mathrm{PhD}$ thesis, Technische Universität München, Germany, October 2004.

24. A. Stamatakis, "Phylogenetic Models of Rate Heterogeneity: A High Performance Computing Perspective," in Proc. of
IPDPS2006, HICOMB Workshop, Proceedings on CD, Rhodos, Greece, April 2006.

25. A. Stamatakis, "RAxML-VI-HPC: Maximum Likelihoodbased Phylogenetic Analyses with Thousands of Taxa and Mixed Models," Bioinformatics, vol. 22, no. 21, 2006, pp. 2688-2690.

26. A. Stamatakis, T. Ludwig, and H. Meier, "Parallel Inference of a 10.000-taxon Phylogeny with Maximum Likelihood," in Proc. of Euro-Par 2004, September 2004, pp. 997-1004.

27. A. Stamatakis, T. Ludwig, and H. Meier, "RAxML-III: A Fast Program for Maximum Likelihood-based Inference of Large Phylogenetic Trees," Bioinformatics, vol. 21, no. 4, 2005, pp. 456-463.

28. A. Stamatakis, M. Ott, and T. Ludwig, "RAxML-OMP: An Efficient Program for Phylogenetic Inference on SMPs," PaCT, 2005, pp. 288-302.

29. C. Stewart, D. Hart, D. Berry, G. Olsen, E. Wernert, and W. Fischer, "Parallel Implementation and Performance of FastDNAml-A Program for Maximum Likelihood Phylogenetic Inference," in Proc. of SC2001, Denver, CO, November 2001.

30. D. Wang, "Cell Microprocessor III," Real World Technologies, July 2005.

31. D. Zwickl, Genetic Algorithm Approaches for the Phylogenetic Analysis of Large Biologiical Sequence Datasets under the Maximum Likelihood Criterion. $\mathrm{PhD}$ thesis, University of Texas at Austin, April 2006.

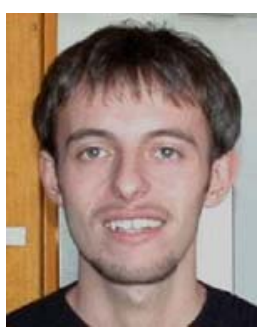

Dr. Alexandros Stamatakis received his Diploma in Computer Science in March 2001 from the Technical University of Munich. His studies included internships at the Ecole Normale Superieure de Lyon, France (9 months), at the Eurocontrol Experimental Center near Paris, France (4 months) and at the Instituto de Salud Carlos III/Universidad Politecnica de Madrid, Spain (3 months). In October 2004 he received his $\mathrm{PhD}$ for research on "Distributed and Parallel Algorithms and Systems for Inference of Huge Phylogenetic Trees based on the Maximum Likelihood Method" from the Technical University of Munich. From January 2005 to June 2006 he worked as postdoctoral researcher at the Institute of Computer Science in Heraklion, Greece. In July 2006 he joined the Laboratory for Computational Biology and Bioinformatics at the Swiss Federal Institute of Technology at Lausanne, Switzerland as a PostDoc. His main research 
interests are technical and algorithmic solutions for inference of huge phylogenetic trees and compute-intensive problems in Bioinformatics.

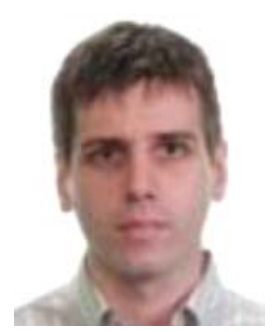

Filip Blagojevic is a $\mathrm{PhD}$ student at the Computer Science department at Virginia Tech. He received his BS degree in Mathematics from University of Belgrade (2002) and his MS Degree in Computer Science from the College of William and Mary (2005). His research interests include, but are not limited to the following: process scheduling, memory management, performance modeling, emerging architectures: CMP- and SMT-based Multiprocessors and IBM/Sony/Toshiba CELL processor.

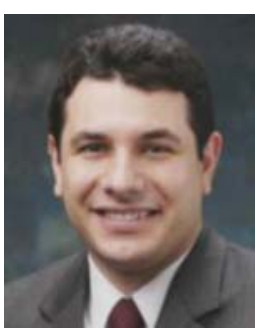

Dimitris Nikolopoulos is an Associate Professor of Computer Science at the Virginia Polytechnic Institute and State University (Virginia Tech) since August 2006. Prior to joining Virginia Tech, he has held a tenure-track faculty position at the College of William and Mary and a visiting faculty position at the University of Illinois at Urbana-Champaign. Nikolopoulos is a recipient of an NSF CAREER award in 2004, a DOE CAREER award in 2005, an IBM Faculty Award in 2007, and six best paper awards, including awards from the ACM SIGPLAN Conference on Principles and Practice of Parallel Programming (PPoPP), the Supercomputing Conference (SC) and the International Parallel and Distributed Processing Symposium (IPDPS). Nikolopoulos conducts research on high-end and embedded multi-processor architectures. His current research activities focus on the interface between multi-core architectures and system software. His contributions appear in more than 75 authored or co-authored papers in the leading journals and conferences in the field of parallel computing and architectures. Nikolopoulos holds a Ph.D. in Computer Engineering (2000) and a Diploma in Computer Engineering (1996), both from the University of Patras. He is a member of ACM, IEEE, and the Technical Chamber of Greece.

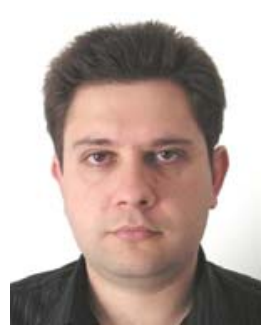

Dr. Christos Antonopoulos received his Diploma in Computer Engineering \& Informatics, MSc and $\mathrm{PhD}$ from the Computer Engineering \& Informatics Department of the University of Patras, Greece. Following, he spent 2 years in U.S. as a Post-Doctoral Research Associate at the College of William \& Mary. He currently serves as Visiting Faculty at the Computer and Communications Engineering Department of the University of Thessaly in Volos, Greece. His research interests include high performance computing architectures, hybrid architectures based on SMT or CMP processors, system software, continuous performance-driven run-time optimizations, memory management, and power management with software techniques. He has been actively involved in many national, EU and US research projects. 